\title{
Fatores intervenientes na terapia fonoaudiológica de crianças autistas
}

\section{Intervening factors in language therapy with autistic children}

\author{
Cibelle Albuquerque de La Higuera Amatoํ, Daniela Regina Molini-Avejonas ${ }^{2}$, Thais Helena Ferreira Santos ${ }^{3}$, \\ Ana Gabriela Lopes Pimentel ${ }^{4}$, Vivian de Campos Valino ${ }^{5}$, Fernanda Dreux Miranda Fernandes ${ }^{6}$
}

\begin{abstract}
RESUMO
Estudos recentes mencionam que a incidência dos distúrbios do espectro autístico chega a 1\%. Isso implica na necessidade de identificação urgente de modelos de intervenção eficazes, bem como dos fatores que podem interferir nesses processos. O objetivo deste artigo é descrever três anos de processos de terapia de linguagem de três crianças com diagnósticos incluídos no espectro do autismo com diferentes características de desenvolvimento e diferentes respostas ao processo terapêutico. Todas as crianças são atendidas em sistema ambulatorial, uma vez por semana, num serviço especializado, por fonoaudiólogas pós-graduandas na área há aproximadamente seis meses antes dos primeiros relatos apresentados. Os casos apresentados evidenciam a diversidade do fenótipo do autismo. Embora não fosse o objetivo deste estudo, fica aparente a referencia a três diferentes quadros incluídos no espectro do autismo. Desta forma, os processos de intervenção foram objeto de sutis ajustes às necessidades e possibilidades de cada uma das crianças. Todas as crianças tiveram progressos importantes em suas manifestações. A análise longitudinal individualizada de processos de intervenção terapêutica permite a abordagem de aspectos associados que podem ser determinantes nos resultados e que exigem abordagem consistente.
\end{abstract}

Descritores: Transtorno autístico; Criança; Transtornos da linguagem/terapia; Fonoterapia/métodos; Terapêutica

\section{INTRODUÇÃO}

Estudos recentes mencionam que a incidência dos distúrbios do espectro autístico (DEA) chega a um por cento ${ }^{(1)}$. Isso

Trabalho realizado no Laboratório de Investigação Fonoaudiológica nos Distúrbios do Espectro Autístico do Departamento de Fisioterapia, Fonoaudiologia e Terapia Ocupacional da Faculdade de Medicina, Universidade de São Paulo - USP - São Paulo (SP), Brasil.

(1) Departamento de Fisioterapia, Fonoaudiologia e Terapia Ocupacional da Faculdade de Medicina, Universidade de São Paulo - USP - São Paulo (SP), Brasil.

(2) Departamento de Fisioterapia, Fonoaudiologia e Terapia Ocupacional da Faculdade de Medicina, Universidade de São Paulo - USP - São Paulo (SP), Brasil.

(3) Programa de Pós-graduação (Mestrado) em Ciências da Reabilitação do Departamento de Fisioterapia, Fonoaudiologia e Terapia Ocupacional da Faculdade de Medicina, Universidade de São Paulo - USP - São Paulo (SP), Brasil.

(4) Programa de Pós-graduação (Mestrado) em Ciências da Reabilitação do Departamento de Fisioterapia, Fonoaudiologia e Terapia Ocupacional da Faculdade de Medicina da Universidade de São Paulo - USP - São Paulo (SP), Brasil.

(5) Programa de Pós-graduação (Mestrado) em Ciências da Reabilitação do Departamento de Fisioterapia, Fonoaudiologia e Terapia Ocupacional da Faculdade de Medicina, Universidade de São Paulo - USP - São Paulo (SP), Brasil. (6) Departamento de Fisioterapia, Fonoaudiologia e Terapia Ocupacional da Faculdade de Medicina, Universidade de São Paulo - USP - São Paulo (SP), Brasil.

Endereço para correspondência: Fernanda Dreux Miranda Fernandes. R. Cipotânea, 51, Cidade Universitária, São Paulo, (SP), Brasil, CEP: 05360-160.

E-mail: fernandadreux@usp.br

Recebido em: 03/12/2010; Aceito em: 11/01/2011 implica na necessidade de identificação urgente de modelos de intervenção eficazes, bem como dos fatores que podem interferir nesses processos ${ }^{(2)}$.

A distinção entre os diversos quadros que compõem o espectro do autismo não está completamente definida ${ }^{(3)}$, mas já há um consenso bastante claro no que diz respeito à existência de um espectro ${ }^{(4)}$.

De qualquer forma, a questão do diagnóstico envolve, desde a distinção entre a suspeita de surdez por algumas famílias ${ }^{(5)}$, associada à ausência de linguagem de algumas crianças autistas, até as discussões a respeito dos limites que podem ser determinados entre os grandes distúrbios do desenvolvimento, como os do espectro do autismo, os distúrbios específicos de linguagem e os transtornos de hiperatividade e déficit de atenção ${ }^{(6)}$.

As famílias com crianças autistas têm sido objeto de diversos estudos encontrados na literatura internacional, como pode ser visto em recente revisão ${ }^{(7)}$. Em nossa realidade, os estudos enfocam também a qualidade de vida das famílias com crianças com $D_{E A}^{(8)}$ e a sua participação nos processos de diagnóstico, contribuindo com o fornecimento de dados a respeito do desempenho das crianças ${ }^{(9)}$.

Aspectos específicos da realidade nacional têm sido abordados em estudos a respeito de processos cada vez mais cuidadosos para o diagnóstico fonoaudiológico das crianças com $\mathrm{DEA}^{(10)}$, os resultados dos processos de inclusão de crianças 
com DEA no ensino regular ${ }^{(11)}$ e resultados de processos de terapia fonoaudiológica de longo termo ${ }^{(12)}$.

Relatos sistematizados a respeito de processos terapêuticos podem contribuir ${ }^{(13,14)}$ para a construção de um corpo de evidencias que fundamente a tomada de decisões a respeito de propostas de intervenção direcionadas a crianças com DEA.

Este é o objetivo deste artigo: descrever três anos de processos de terapia de linguagem de três crianças com diagnósticos incluídos no espectro do autismo, com diferentes características de desenvolvimento e diferentes respostas ao processo terapêutico. Todas as crianças são atendidas em sistema ambulatorial, uma vez por semana, num serviço especializado, por fonoaudiólogas pós-graduandas na área e os responsáveis assinaram o termo de consentimento que autoriza o registro e a divulgação dos resultados dos processos terapêuticos (processo número 460/02). O atendimento fonoaudiológico foi iniciado aproximadamente seis meses antes dos primeiros relatos aqui apresentados.

\section{APRESENTAÇÃO DOS CASOS CLÍNICOS}

\section{Caso 1}

Menina com diagnóstico de autismo e seis anos de idade no início do atendimento fonoaudiológico, frequentava préescola do ensino publico regular. É trazida para a terapia sempre por sua mãe, mas apresenta taxa de ausências próxima dos $50 \%$.

\section{Comportamento, socialização e interesses:}

2007 - manipula revistas e livros; traz folhas de papel para o atendimento, mas limita-se a folheá-las. Muito agitada, permanecendo apenas alguns instantes em cada local da sala ou com algum material. No segundo semestre começa a mostrar mais interesse por objetos de casa (brinquedos e miniaturas). A professora relata episódios de agressividade na escola.

2008 - mantém o interesse por papeis, mas passa a brincar com miniaturas, executando atividades de seriação e de imitação diferida e no segundo semestre passa a montar quebra-cabeças; mas mantém o tempo de atenção reduzido, embora com menos agitação. No início do ano recusa-se a sair da sala para ir embora, jogando-se no chão, mas a partir do segundo semestre estabelece uma relação amistosa com a terapeuta. Mantém trocas interativas e ao longo do ano passa a aceitar e pedir contato físico (despede-se com um beijinho).

2009 - faz contato ocular sistemático, mantém o reduzido tempo de atenção e interesse por alguma atividade. Monta quebra-cabeças, faz jogo simbólico com atividades relacionadas ao cotidiano. Durante essas atividades engajase em situações de atenção compartilhada com a iniciativa da terapeuta.

\section{Comunicação e linguagem:}

2007 - comunica-se preferencialmente pelo meio gestual em atividades de interação e regulação. Faz alguma troca de turnos comunicativos, mas suas emissões caracterizam-se como vocalizações devido à grande distorção articulatória, que compromete sua inteligibilidade.

2008 - continua a se comunicar preferencialmente pelo meio gestual, mas passa a usar os meios vocal e verbal com mais freqüência. Apesar da imprecisão articulatória, fica evidente a limitação do vocabulário. Passa a evidenciar mais intenção comunicativa.

2009 - embora continue a haver alguma imprecisão articulatória e os meios comunicativos preferenciais continuem a ser, respectivamente, o gestual e o vocal, fica evidente o aumento da proporção da comunicação direcionada ao interlocutor, especialmente o jogo compartilhado.

O Quadro 1 mostra o resultado observado nas diversas áreas do Desempenho Sócio-Cognitivo das três crianças testadas ao longo dos três anos.

A Figura 1 ilustra a evolução de aspectos funcionais da comunicação.

\section{Caso 2}

Menino com diagnóstico de autismo e oito anos de idade no início do atendimento fonoaudiológico, frequentava a segunda série do ensino publico regular, com relato de boa adaptação. É trazido para a terapia sempre por sua mãe e apresenta menos de 5\% de faltas.

\section{Comportamento, socialização e interesses:}

2007 - criança falante, que inicia turnos com desconhecidos a caminho da sala de terapia, mas não mantém conversações com diversos turnos de comunicação. Faz jogo simbólico elaborado e mantém-se por longo tempo

Quadro 1. Desempenho sócio-cognitivo testado no período de 2007 a 2009 nos três casos

\begin{tabular}{|l|c|c|c|c|c|c|c|c|c|}
\hline \multirow{2}{*}{ Áreas testadas } & \multicolumn{3}{|c|}{2007} & \multicolumn{3}{|c|}{2008} & \multicolumn{3}{c|}{2009} \\
\cline { 2 - 10 } & C1 & C2 & C3 & C1 & C2 & C3 & C1 & C2 & C3 \\
\hline Intenção comunicativa gestual & 4 & 6 & 3 & 6 & 6 & 6 & 6 & 6 & 6 \\
Intenção comunicativa vocal & 4 & 6 & 3 & 5 & 6 & 6 & 5 & 6 & 6 \\
Uso de objeto mediador & 3 & 3 & 3 & 4 & 4 & 3 & 4 & 4 & 4 \\
Imitação gestual & 3 & 4 & 3 & 4 & 4 & 4 & 4 & 4 & 4 \\
Imitação vocal & 4 & 3 & - & 3 & 4 & 2 & 3 & 4 & 4 \\
Jogo combinatório & 6 & 5 & - & 5 & 5 & 3 & 5 & 6 & 5 \\
Jogo simbólico & 6 & 6 & - & 6 & 6 & 6 & 6 & 6 \\
\hline
\end{tabular}

Legenda: $\mathrm{C} 1=$ caso $1 ; \mathrm{C} 2=$ caso $2 ; \mathrm{C} 3=$ caso 3 


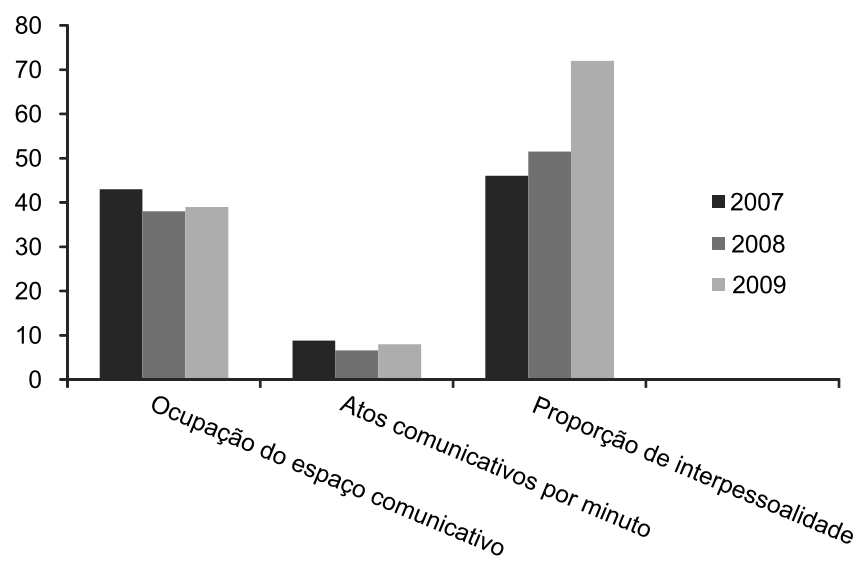

Figura 1. Aspectos funcionais da comunicação (caso 1)

numa mesma atividade, em jogo auto-centrado, raramente engajando-se em atividades de jogo compartilhado. Esporadicamente apresenta manifestações descontextualizadas, como cantar e dançar em situações inapropriadas.

2008 - mantém bom contato com a terapeuta, aceita melhor a participação de outros, parece menos auto-centrado nas brincadeiras, permanecendo menos tempo em uma mesma atividade, mas não toma a iniciativa de propor outras alternativas. Quando está muito envolvido em uma atividade, não aceita a participação da terapeuta. Em geral essas situações envolvem um jogo simbólico complexo e elaborado.

2009 - mantém-se atento e focalizado, mas passa a solicitar a ajuda da terapeuta quando encontra alguma dificuldade, manifesta verbalmente suas intenções e desejos. Suas atividades favoritas passam a incluir fantoches, engajando-se em jogos cooperativos com a terapeuta, participando de longos diálogos com a intermediação de diferentes fantoches.

\section{Comunicação e linguagem:}

2007 - apresenta ecolalia tardia freqüiente em situações de jogo auto-centrado. O meio de comunicação preferencial é o verbal, que usa de forma socialmente apropriada em contatos superficiais, em geral em marcadores de polidez e de reconhecimento do outro, que são basicamente as únicas iniciativas de comunicação espontânea. Embora na maior parte das vezes responda às iniciativas da terapeuta, geralmente o faz com frases de uma só palavra.

2008 - parece compreender melhor a expressão facial e os gestos da terapeuta, mas ainda estabelece pouco contato ocular. Continua a apresentar alguma ecolalia tardia, que parece relacionada a alguma iniciativa de comunicação, como um pedido de rotina social. Apresenta mais iniciativas de comunicação relacionadas a seus interesses, com frases curtas ou palavras isoladas.

2009 - continua apresentando ecolalia tardia em momentos de menor interação, mas inicia turnos de comunicação, reconhece e repara quebras comunicativas, identifica falhas e utiliza recursos para manutenção de atenção do interlocutor. Utiliza alguns gestos, principalmente como apoio para atos comunicativos com função de expressão de protesto.

A Figura 2 ilustra a evolução de aspectos funcionais da comunicação.

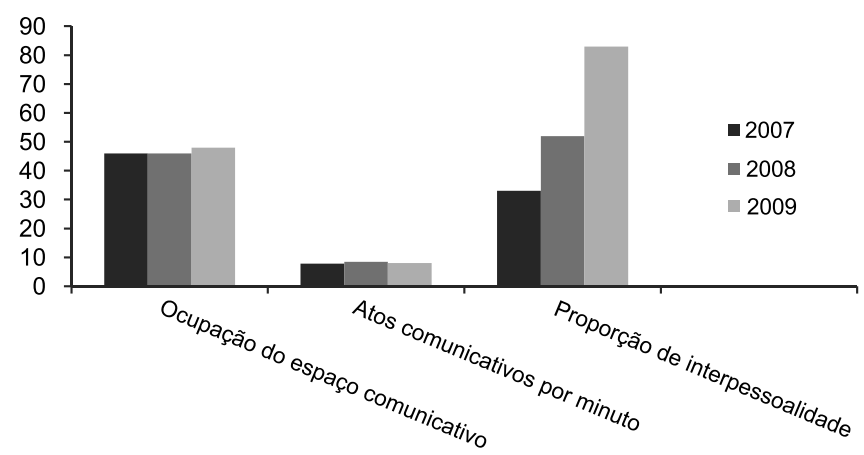

Figura 2. Aspectos funcionais da comunicação (caso 2)

\section{Caso 3}

Menino com diagnóstico de autismo e quatro anos de idade no início do atendimento fonoaudiológico, frequentava uma pré-escola regular com relato de bom desempenho e especial interesse por leitura. É trazido para a terapia sempre por sua mãe e quase não falta ao atendimento.

\section{Comportamento, socialização e interesses:}

2007 - reconhece a terapeuta, mantém contato ocular e utiliza esse recurso para manter a atenção do interlocutor. É resistente a mudanças de rotina e de atividades. Por vezes joga-se no chão no final da terapia, recusando-se a sair e chorando bastante. Faz jogo simbólico e engaja-se em jogos compartilhados. Faz leitura logográfica e interessase por leitura, mas não nomeia formas e cores, embora as reconheça.

2008 - mantém o contato ocular, adaptou-se melhor à rotina e ao tempo da terapia, não mostrando resistência para ir embora. Envolve-se em jogos simbólicos complexos, mas não propõe situações novas durante a brincadeira. Lê algumas palavras

2009 - busca contato físico e estabelece trocas de turno, mas seu contato ocular está menos sistemático e há pouca atenção para as expressões faciais da terapeuta. Desenvolve rotinas, como colocar o pé na parte vermelha dos degraus quando sobe a escada. Evidencia grande interesse pelo computador, mas aceita propostas de outras atividades.

\section{Comunicação e linguagem:}

2007 - comunica-se predominantemente pelos meios verbal e vocal, com o apoio de gestos. Tem alguma imprecisão articulatória, mas aceita repetir ou reformular suas emissões quando a terapeuta solicita. Não evidencia dificuldades de compreensão de linguagem.

2008 - produz frases mais longas e repara as quebras comunicativas quando a terapeuta solicita, mas não reconhece estas quebras sem este auxílio. Suas emissões estão mais precisas, o que contribui para a ampliação da proporção do uso do meio verbal de comunicação.

2009 - demonstra intenção comunicativa, produz frases com estrutura sintática adequada e compreende ordens simples e complexas. Inicia turnos, introduz tópicos e mantém o diálogo quando interessado. Utiliza os gestos de apoio de forma mais consistente, o que contribui para o aumento 
na proporção do uso desse meio comunicativo. Não produz narrativas coerentes.

A Figura 3 ilustra a evolução de aspectos funcionais da comunicação.

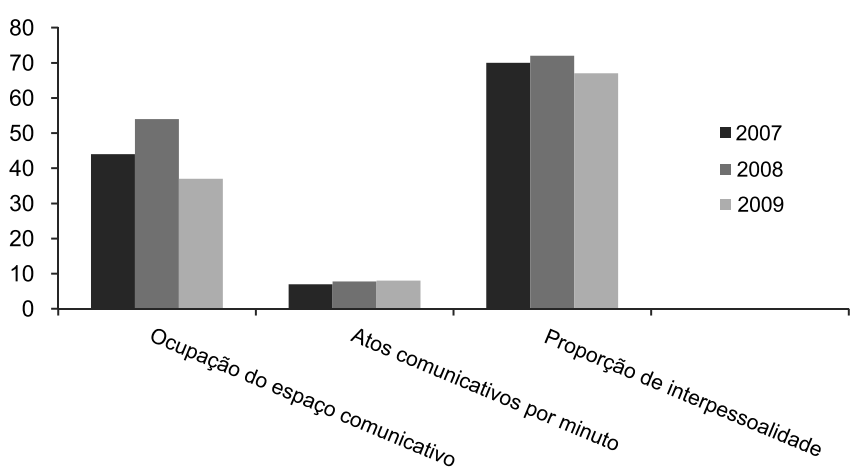

Figura 3. Aspectos funcionais da comunicação (caso 3)

\section{DISCUSSÃO}

As três crianças foram atendidas em esquema ambulatorial, uma vez por semana, em terapia individual durante todo o período envolvido por esse estudo. A cada ano há dois períodos de férias que têm a duração de duas semanas em julho e três semanas entre o final de dezembro e o início de janeiro. Embora haja a troca anual de terapeutas no início de cada ano (nos casos deste estudo isso ocorreu em março, pois é o período de substituição dos alunos de pós-graduação) os processos têm sua continuidade garantida a partir de reavaliações constantes, manutenção dos mesmos supervisores e acompanhamento intensivo de cada caso.

Os casos apresentados evidenciam a diversidade do fenótipo do autismo ${ }^{(2,4)}$, pois temos uma menina de seis anos, com grandes dificuldades de comportamento, muito poucas emissões inteligíveis e uma gama restrita de interesses não funcionais; o primeiro menino descrito tem oito anos de idade, bom desempenho na escola, à qual está adaptado, mas com dificuldades de empatia, pouca iniciativa de comunicação, manifestações descontextualizadas e ecolalia; por fim, o segundo menino apresentado tem quatro anos, desempenho cognitivo acima do esperado para a idade, interesses incompatíveis para a idade, muita dificuldade de adaptação social e facilidade com os aspectos formais da linguagem.

Embora não fosse o objetivo deste estudo, fica aparente a referência a três diferentes quadros incluídos no espectro do autismo $^{(3,4,6)}$. Desta forma, os processos de intervenção foram objeto de sutis ajustes às necessidades e possibilidades de cada uma das crianças $^{(2,9,13,14)}$.

É interessante observar que todas as crianças tiveram progressos importantes em suas manifestações. A menina passa a engajar-se em atividades de atenção compartilhada e jogo simbólico e, aumenta significativamente a proporção de comunicação interpessoal. O primeiro menino passa a envolver-se em jogos compartilhados, diálogos e trocas interpessoais, inicia turnos comunicativos, utiliza recursos discursivos de forma adequada e usa comunicação não verbal como apoio. O segundo menino mostra grande interesse pelo computador, mas aceita alternar sua utilização com atividades mais interativas, usa gestos de apoio de forma consistente, demonstra habilidades sintáticas e discursivas adequadas, embora não apresente narrativas produtivas.

Esses resultados demandam a consideração dos fatores intervenientes em cada processo ${ }^{(2,13,14)}$. Como observado anteriormente, a menina não compareceu a praticamente a metade dos atendimentos planejados e mesmo assim seu progresso foi significativo. Esse resultado exige a reflexão a respeito do que teria acontecido se ela tivesse frequientado todas as sessões propostas e quais as alternativas para a gestão do processo terapêutico em casos como este. É comum que as ausências à terapia sejam justificadas por fatores objetivos e concretos, em realidades complexas como as que se encontra na cidade de São Paulo ${ }^{(8)}$; no entanto, a avaliação do investimento representado pela frequência, mesmo que assistemática, e pela reserva de horário para um paciente pouco frequiente exige dados objetivos para fundamentar as decisões. O observado neste caso é que, mesmo com um grande número de ausências, a criança aproveitou o processo terapêutico.

O primeiro menino nos revela uma situação praticamente oposta. Família e escola colaboradoras e interessadas e a possibilidade de atendimento clínico apenas uma vez por semana (por questões de espaço físico inerentes ao ambulatório) demandam reflexões a respeito das possibilidades de ampliação dessa oferta. Trata-se de uma criança que, provavelmente devido ao tipo de manifestação apresentada, foi trazida para atendimento especializado com mais de seis anos de idade, quando há relatos consistentes de melhores progressos em crianças que recebem intervenção adequada mais precocemente ${ }^{(14)}$. Uma forma de compensar esse atraso seria a oferta de um programa de intervenção mais intensivo, que não tem sido acessível a todas as crianças em nosso meio.

O segundo menino, por outro lado, desperta interesse tanto nos membros da família quanto na escola, devido ao seu interesse por leitura e pelos aspectos formais da linguagem, o que dá a impressão de um funcionamento acima da média. Isso tem levado ao aumento da disponibilização de atividades e materiais que reforçam o interesse e a manutenção de atividades de sistematização, em detrimento das que incluem empatia. Isso evidencia a necessidade de maiores investimentos em ações dirigidas à orientação familiar e escolar ${ }^{(11,12)}$.

\section{COMENTÁRIOS FINAIS}

A análise longitudinal individualizada de processos de intervenção terapêutica permite a abordagem de aspectos associados que podem ser determinantes nos resultados e que exigem abordagem consistente.

O registro sistemático desses processos terapêuticos é fundamental para que experiências individuais possam ser analisadas de forma significativa para contribuir para a prática baseada em evidência.

No que se refere ao espectro do autismo, considerandose a incidência revelada, é fundamental que as experiências individuais e com pequenos grupos sejam sistematizadas no sentido de obter alternativas para a abordagem de um número muito grande de crianças que provavelmente apresentam a mesmas necessidades, mas que não foram diagnosticadas ou não encontraram o atendimento especializado. 


\begin{abstract}
Recent studies state that the incidence of autism spectrum disorders is $1 \%$ of the infantile population. It implies the need of urgent identification of efficient intervention proposals as well as of the factors that may intervene in these processes. The aim of this study is to describe three years of language therapy processes of three children diagnosed within the autism spectrum with different developmental characteristics and different responses to the therapeutic process. All the children were attending a specialized ambulatory program once a week. The language therapists were post-graduate students in the field and the therapeutic intervention started approximately six months prior to the beginning of the study. The children presented evidence of the diversity of the autism phenotype. Although it was not the purpose of this study, the reference to the three different features of the autism spectrum is clear. This way, the intervention processes received subtle adjustments to each child's specific needs and possibilities. All children had significant progress in their manifestations. The longitudinal individual analysis of therapeutic intervention processes allows the identification of associate process that might be determinant to the results and that demand consistent approaches.
\end{abstract}

Keywords: Autistic disorder; Child; Language disorders; Speech therapy/methods; Therapeutics

\title{
REFERÊNCIAS
}

1. Baird G, Simonoff E, Pickles A, Chandler S, Loucas T, Meldrum $\mathrm{D}$, Charman T. Prevalence of disorders of the autism spectrum in a population cohort of children in South Thames: the Special Needs and Autism Project (SNAP). Lancet. 2006; 368(9531):210- 5.

2. Charman T. Developmental approaches to understanding and treating autism. Folia Phoniatr Logop. 2010;62(4): 166-77.

3. Tamanaha AC, Perissinoto J, Chiari BM. Uma breve revisão histórica sobre a construção dos conceitos do autismo infantil e da síndrome de Asperger. Rev Soc Bras Fonoaudiol. 2008; 13(3): 296-9.

4. Lord C, Risi S. Diagnosis of autism spectrum disorders in young children. In: Wetherby AM, Prizant BM, editors. Autism spectrum disorders - A yransactional developmental perspective. Baltimore: Paul Brooks; 2001. p.11-30.

5. Sousa EC, Lima FT, Tamanaha A, Perissinoto J, Azevedo MF, Chiari BM. A associação entre a suspeita inicial de perda auditiva e a ausência de comunicação em crianças com transtorno do espectro autístico. Rev Soc Bras Fonoaudiol. 2009; 14(3): 487-90.

6. Befi-Lopes DM, Cáceres AM. Refletindo sobre o Novo: perfis lingüísticos em distúrbios do espectro autístico, distúrbios específico de linguagem e transtorno de hiperatividade e déficit de atenção. Rev Soc Bras Fonoaudiol. 2010; 15(2): 305-6.

7. Fernandes FD. Famílias com crianças autistas na literatura internacional. Rev Soc Bras Fonoaudiol. 2009; 14(3): 427-32.
8. Barbosa MR, Fernandes FD. Qualidade de vida dos cuidadores de crianças com transtorno do espectro autístico. Rev Soc Bras Fonoaudiol. 2009; 14(3): 482-6.

9. Sousa-Morato PF, Fernandes FD. Adaptação sócio-comunicativa no espectro autístico: dados obtidos com pais e terapeutas. Rev Soc Bras Fonoaudiol. 2009; 14(2): 225-33.

10. Moreira CR, Fernandes FD. Avaliação da comunicação no espectro autístico: interferência da familiaridade no desempenho de linguagem. Rev Soc Bras Fonoaudiol. 2010; 15(3): 430-5.

11. Brito MC, Carrara K. Alunos com distúrbios do espectro autístico em interação com professores na educação inclusiva: descrição de habilidades pragmáticas. Rev Soc Bras Fonoaudiol. 2010; 15(3): 421-9.

12. Misquiatti AR, Brito MC. Terapia de linguagem de irmãos com transtornos invasivos do desenvolvimento: estudo longitudinal. Rev Soc Bras Fonoaudiol. 2010; 15(1): 134-9.

13. Keen D, Rodger S, Doussin K, Braithwaite M. A pilot study of the effects of a social-pragmatic intervention on the communication and symbolic play of children with autism. Autism. 2007; 11(1): 63-71.

14. Sutera A, Pandey J, Esser EL, Rosenthal MA, Wilson LB, Barton M, et al. Predictors of optimal outcome in toddlers diagnosed with autism spectrum disorders. J Autism Dev Disord. 2007; 37(1):98-107. 